\title{
Exclusion of Leptophilic Dark Matter Models using XENON100 Electronic Recoil Data
}

\author{
E. Aprile,${ }^{1}$ F. Agostini, ${ }^{2,}$ M. Alfonsi,${ }^{4}$ L. Arazi,${ }^{5}$ K. Arisaka, ${ }^{6}$ F. Arneodo,${ }^{7}$ M. Auger,${ }^{8}$ C. Balan, ${ }^{9}$ \\ P. Barrow, ${ }^{8}$ L. Baudis,${ }^{8}$ B. Bauermeister, ${ }^{10}$ A. Behrens, ${ }^{8}$ A. Brown,${ }^{11,4}$ E. Brown, ${ }^{12}$ S. Bruenner, ${ }^{13}$ \\ G. Bruno, ${ }^{14}$ R. Budnik, ${ }^{5}$ L. Bütikofer,${ }^{15}$ J. M. R. Cardoso,${ }^{9}$ M. Cervantes,${ }^{11,}{ }^{*}$ D. Coderre,${ }^{15}$ A. P. Colijn,${ }^{4}$ \\ H. Contreras, ${ }^{1}$ J. P. Cussonneau, ${ }^{16}$ M. P. Decowski, ${ }^{4}$ A. Di Giovanni, ${ }^{7}$ E. Duchovni, ${ }^{5}$ S. Fattori,${ }^{10}$ \\ A. D. Ferella, ${ }^{2}$ A. Fieguth,,${ }^{14}$ W. Fulgione,${ }^{2}$ F. Gao,${ }^{17}$ M. Garbini,${ }^{3}$ C. Geis,${ }^{10}$ L. W. Goetzke, ${ }^{1}$ C. Grignon, ${ }^{10}$ \\ E. Gross ${ }^{5}$ W. Hampel,${ }^{13}$ R. Itay,${ }^{5}$ F. Kaether ${ }^{13}$ B. Kaminsky, ${ }^{15}$ G. Kessler ${ }^{8}$ A. Kish,${ }^{8}$ H. Landsman, ${ }^{5}$ \\ R. F. Lang, ${ }^{11}{ }^{\dagger}$ M. Le Calloch, ${ }^{16}$ D. Lellouch,${ }^{5}$ L. Levinson, ${ }^{5}$ C. Levy,${ }^{12}$ S. Lindemann, ${ }^{13}$ M. Lindner, ${ }^{13}$ \\ J. A. M. Lopes,${ }^{9}{ }^{\ddagger}$ A. Lyashenko, ${ }^{6}$ S. Macmullin, ${ }^{11}$ T. Marrodán Undagoitia, ${ }^{13}$ J. Masbou, ${ }^{16}$ F. V. Massoli, ${ }^{3}$ \\ D. Mayani Paras, ${ }^{8}$ A. J. Melgarejo Fernandez,${ }^{1}$ Y. Meng, ${ }^{6}$ M. Messina,${ }^{1}$ B. Miguez ${ }^{18}$ A. Molinario, ${ }^{18}$ \\ G. Morana,${ }^{3}$ M. Murra, ${ }^{14}$ J. Naganoma,${ }^{19}$ K. Ni,${ }^{17}$ U. Oberlack, ${ }^{10}$ S. E. A. Orrigo $,{ }^{9},{ }^{\S}$ P. Pakarha,${ }^{8}$ \\ E. Pantic, ${ }^{6}$ R. Persiani,${ }^{3}$ F. Piastra,${ }^{8}$ J. Pienaar,${ }^{11}$ G. Plante,${ }^{1}$ N. Priel,${ }^{5}$ L. Rauch,${ }^{13}$ S. Reichard,${ }^{11}$ \\ C. Reuter, ${ }^{11}$ A. Rizzo, ${ }^{1}$ S. Rosendahl,${ }^{14}$ J. M. F. $\operatorname{dos}$ Santos,${ }^{9}$ G. Sartorelli ${ }^{3}$ S. Schindler, ${ }^{10}$ J. Schreiner, ${ }^{13}$ \\ M. Schumann, ${ }^{15}$ L. Scotto Lavina, ${ }^{16}$ M. Selvi, ${ }^{3}$ P. Shagin,${ }^{19}$ H. Simgen, ${ }^{13}$ A. Teymourian, ${ }^{6}$ D. Thers,${ }^{16}$ \\ A. Tiseni, ${ }^{4}$ G. Trinchero, ${ }^{18}$ C. Tunnell,${ }^{4}$ O. Vitells,${ }^{5}$ R. Wall,${ }^{19}$ H. Wang, ${ }^{6}$ M. Weber, ${ }^{1}$ and C. Weinheimer ${ }^{14}$ \\ (The XENON Collaboration) \\ ${ }^{1}$ Physics Department, Columbia University, New York, NY, USA \\ ${ }^{2}$ INFN-Laboratori Nazionali del Gran Sasso and Gran Sasso Science Institute, L'Aquila, Italy \\ ${ }^{3}$ Department of Physics and Astrophysics, University of Bologna and INFN-Bologna, Bologna, Italy \\ ${ }^{4}$ Nikhef and the University of Amsterdam, Science Park, Amsterdam, Netherlands \\ ${ }^{5}$ Department of Particle Physics and Astrophysics, Weizmann Institute of Science, Rehovot, Israel \\ ${ }^{6}$ Physics \& Astronomy Department, University of California, Los Angeles, CA, USA \\ ${ }^{7}$ New York University Abu Dhabi, Abu Dhabi, United Arab Emirates \\ ${ }^{8}$ Physik-Institut, University of Zurich, Zurich, Switzerland \\ ${ }^{9}$ Department of Physics, University of Coimbra, Coimbra, Portugal \\ ${ }^{10}$ Institut für Physik ${ }^{3}$ Exzellenzcluster PRISMA, Johannes Gutenberg-Universität Mainz, Mainz, Germany \\ ${ }^{11}$ Department of Physics and Astronomy, Purdue University, West Lafayette, IN, USA \\ ${ }^{12}$ Department of Physics, Applied Physics and Astronomy, Rensselaer Polytechnic Institute, Troy, NY, USA \\ ${ }^{13}$ Max-Planck-Institut für Kernphysik, Heidelberg, Germany \\ ${ }^{14}$ Institut für Kernphysik, Wilhelms-Universität Münster, Münster, Germany \\ ${ }^{15}$ Albert Einstein Center for Fundamental Physics, University of Bern, Bern, Switzerland \\ ${ }^{16}$ SUBATECH, Ecole des Mines de Nantes, CNRS/In2p3, Université de Nantes, Nantes, France \\ ${ }^{17}$ Department of Physics 83 Astronomy, Shanghai Jiao Tong University, Shanghai, China \\ ${ }^{18}$ INFN-Torino and Osservatorio Astrofisico di Torino, Torino, Italy \\ ${ }^{19}$ Department of Physics and Astronomy, Rice University, Houston, TX, USA
}

\begin{abstract}
Laboratory experiments searching for galactic dark matter particles scattering off nuclei have so far not been able to establish a discovery. We use data from the XENON100 experiment to search for dark matter interacting with electrons. With no evidence for a signal above the low background of our experiment, we exclude a variety of representative dark matter models that would induce electronic recoils. For axial-vector couplings to electrons, we exclude cross-sections above $6 \times 10^{-35} \mathrm{~cm}^{2}$ for particle masses of $m_{\chi}=2 \mathrm{GeV} / \mathrm{c}^{2}$. Independent of the dark matter halo, we exclude leptophilic models as explanation for the long-standing DAMA/LIBRA signal, such as couplings to electrons through axial-vector interactions at a $4.4 \sigma$ confidence level, mirror dark matter at $3.6 \sigma$, and luminous dark matter at $4.6 \sigma$.
\end{abstract}

Dark Matter in the form of Weakly Interacting Massive Particles (WIMPs) is typically expected to induce nuclear recoils in a terrestrial detector target [1] with an annually modulated rate due to the motion of the Earth around the Sun $[2,3]$. Although such a modulation has been observed by the DAMA/LIBRA collaboration using sodium iodine [4], it is difficult to interpret it as a dark matter signal, given the null results from other experiments [5-7]. In fact, dark matter-induced nuclear recoils are excluded by these results unless one invokes models that are fine-tuned to create a signal only in DAMA/LIBRA but not in other experiments [8-10]. In contrast, dark matter-induced electronic recoils appear as a viable explanation for the observed modulation because exclusions of other experiments do not apply directly in this case [11-13]. We use data from the XENON100 detector to rule out this possibility for three different, representative dark matter models. 


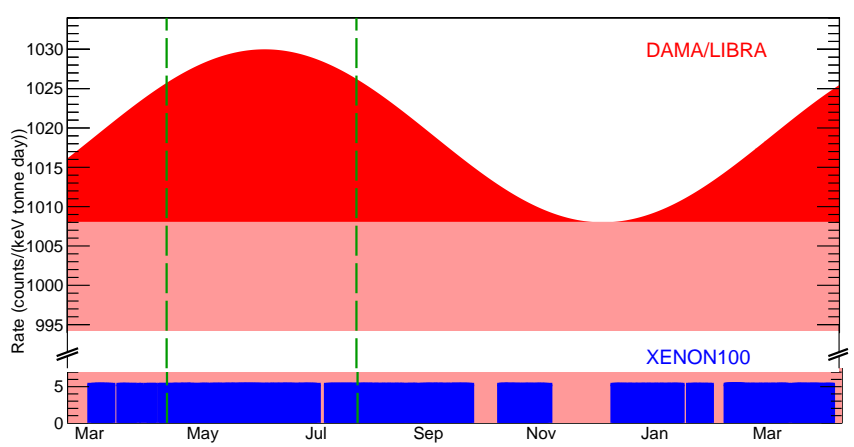

FIG. 1: Fig. 1. Conceptual illustration of the analysis. Shown is the DAMA/LIBRA rate (red) [20] with the modulated rate in $(2-6) \mathrm{keV}$ from the fit parameters in [4] (dark red). The distribution of the XENON100 live time (blue) is indicated with its average background rate of 5.3 events /( $\mathrm{keV} \cdot$ tonne $\cdot$ day), which shows dents due to maintenance or calibration campaigns. The region between the dashed lines (green) indicates the 70 summer live days where the modulated signal is expected to be largest.

We interpret data from the XENON100 detector that were acquired between February 28, 2011 and March 31, 2012 for a total exposure of 224.6 live days and $34 \mathrm{~kg}$ fiducial mass. We have previously searched this data set for spin-independent [14] and spin-dependent [15] WIMPinduced nuclear recoils as well as for axion-induced electronic recoils [16]. XENON100 is located in the Gran Sasso underground laboratory. It consists of a liquid xenon target that is operated as a low-background time projection chamber [17]. Each particle interaction results in two signals: The prompt scintillation signal (S1) is used here for energy estimation, and the delayed ionization signal (S2) allows for 3D vertex reconstruction. Data reduction is performed in order to select single-scatter low-energy $(<10 \mathrm{keV})$ recoils in the fiducial volume, while retaining maximal detector efficiency $[16,18]$. At low energies, the remaining background of XENON100 is dominated by forward-scattered Compton events, resulting in a flat spectrum with a rate of 5.3 events $/(\mathrm{keV} \cdot$ tonne $\cdot$ day $)$ in the fiducial volume [19] (File A1). This rate is more than two orders of magnitude lower than the average background rate of about 1019 events / $(\mathrm{keV} \cdot$ tonne day) reported by DAMA/LIBRA in the same energy interval [20,21], and even smaller than their reported annual modulation amplitude of (11.2 \pm 1.2$)$ events/(keV · tonne · day) [4]. Because the DAMA/LIBRA collaboration has not published the composition of their background at low energies, we test the minimum dark matter signal that would be required to cause the observed modulation. In this scenario, the constant spectrum is fully attributed to background, and only the modulated part itself is attributed to a $100 \%$ modulated dark matter signal as illustrated in (Fig. 1). We ignore the practical difficulties of realizing such a highly modulated signal $[3,22]$ but conservatively consider it as the case that is most challenging to exclude. The dark matter-induced rate would then be zero on December 2nd, and twice the measured modulation amplitude on June 2nd. It follows that there is an optimized time interval to consider for best sensitivity. To find this interval, the signal expected in XENON100 was simulated for different time intervals centered around June 2nd. We take into account uncertainties from counting statistics in XENON100 and DAMA/LIBRA, as well as the systematic uncertainty from the conversion of $\mathrm{keV}$ energy into S1 [16]. The optimum time interval is found to be 70 live days around June 2nd, roughly corresponding to April 2011-August 2011 (Fig. 1) as indicated. Our expected sensitivity varies by less than $0.1 \sigma$ with changes of this interval of \pm 40 live days. A dedicated analysis of the time stability of XENON100 electron recoil data will be presented elsewhere [23].

WIMP axial-vector coupling to electrons: A relativistic treatment of dark matter-electron scattering shows that $\mathrm{keV}$-scale electronic recoils can only be induced by dark matter particles with masses $m_{\chi} \gtrsim$ $1 \mathrm{GeV} / \mathrm{c}^{2}$ scattering inelastically off electrons with momenta on the order of $\mathrm{MeV} / \mathrm{c}[11,24]$.A qualitatively similar result is obtained by a simple non-relativistic treatment of elastic two-body scattering. As shown in [11], even if the dark matter has tree-level (first-order) interactions only with leptons, loop-induced dark matter-hadron interactions dominate the experimental signatures and make the usual exclusions based on nuclear recoil analyses applicable. Thus, we consider here axial-vector $\vec{A} \otimes \vec{A}$ couplings between dark matter and leptons, since in this case, loop contributions vanish, while the WIMP-electron coupling is not suppressed by additional small factors of velocity $v$ or mass ratio $m_{e} / m_{\chi}$.

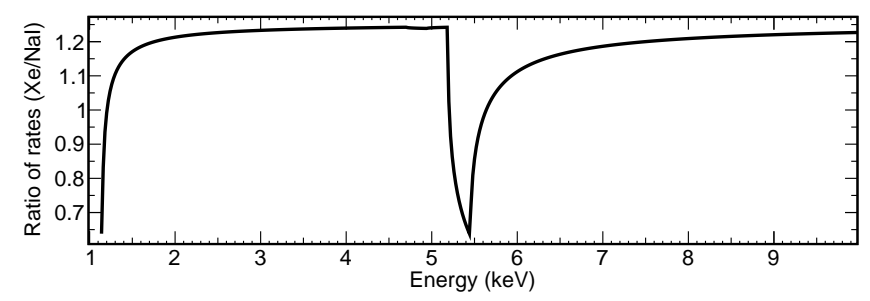

FIG. 2: Fig. 2. Calculated ratio of the differential rates in xenon and sodium iodide for inelastic WIMP-electron scattering through axial-vector coupling. The structures around 1 and $5 \mathrm{keV}$ are owing to the small difference in the binding energies of the $3 s$ and $2 s$ shells in xenon and iodine.

We use equation (30) in [11], with an additional factor of 2 to account for electron occupancy from spin, to calculate the differential rate for WIMP-electron scattering (File A2). The expected rate includes a sum over the atomic shells of the target, and for each shell, integrates the momentum wave function of the electrons to 
get the contribution at a given recoil energy. Given the requirement that the energy deposited in the detector must be more than the binding energy of the electron, the largest contribution to the rate in a sodium iodide target comes from the $3 \mathrm{~s}$ shell of iodine. The contributions from sodium are two orders of magnitude smaller. The momentum-space wave functions for xenon atoms and iodine anions are nearly identical as a result of their similar electron structure. This has the important consequence that a comparison between sodium iodide and xenon is independent of the dark matter halo. The ratio of the calculated differential rates in xenon and sodium iodide are shown in Fig. 2 as a function of deposited energy, considering the full shell structure. This ratio has negligible dependence on the WIMP mass.

We contrast the DAMA/LIBRA signal, interpreted as WIMPs coupling to electrons through axial-vector interactions, with XENON100 data. The energy spectrum of the modulation amplitude [4] is multiplied by the energydependent ratio from Fig. 2 and by a constant factor of 1.88 , which accounts for the time integral of the modulated signal that is expected in our 70 summer live days (Fig. 1). The deposited electronic recoil energy in XENON100 is estimated from the S1 signal, measured in photoelectrons (PE), using the NESTv0.98 model [25] which consistently fits the available data [26-29]. The energy scale, shown in [16], includes a systematic uncertainty that decreases from $20 \%$ to $7 \%$ from $1 \mathrm{keV}$ to $10 \mathrm{keV}$, reflecting the spread and uncertainties in the measurements. The $\mathrm{S} 1$ generation is modelled as a Poisson process and the PMT resolution is taken into account in order to obtain the predicted XENON100 S1 spectrum from the scaled energy spectrum [18]. Our resolution is a factor 2 worse than that of DAMA/LIBRA; the feature at $5.2 \mathrm{keV}$ in Fig. 2 is lost in this process.

The converted DAMA/LIBRA and measured XENON100 energy spectra are shown in Fig. 3. Part of the DAMA/LIBRA signal is expected to be seen below $2 \mathrm{keV}$ due to the finite energy resolution of XENON100. The uncertainty in the converted signal includes both the statistical uncertainty in the original DAMA/LIBRA energy spectrum [4] as well as the uncertainties from our energy conversion. The electronic recoil cut acceptance, shown in [16], was applied to the converted DAMA/LIBRA spectrum. The uncertainty shown in the XENON100 data is statistical.

The energy region to determine the level of exclusion was chosen starting at the threshold of $3 \mathrm{PE}$ [14] to the point where the DAMA/LIBRA signal falls below the expected average XENON100 rate (cyan in Fig.3, calculated using a flat spectrum background model and scaled for the live time of the data set), which is at $14 \mathrm{PE}$, corresponding to (2.0-5.9) keV. Taking systematic uncertainties into account, a simple comparison of the integral counts in this energy interval excludes the DAMA/LIBRA signal as axial-vector coupling between

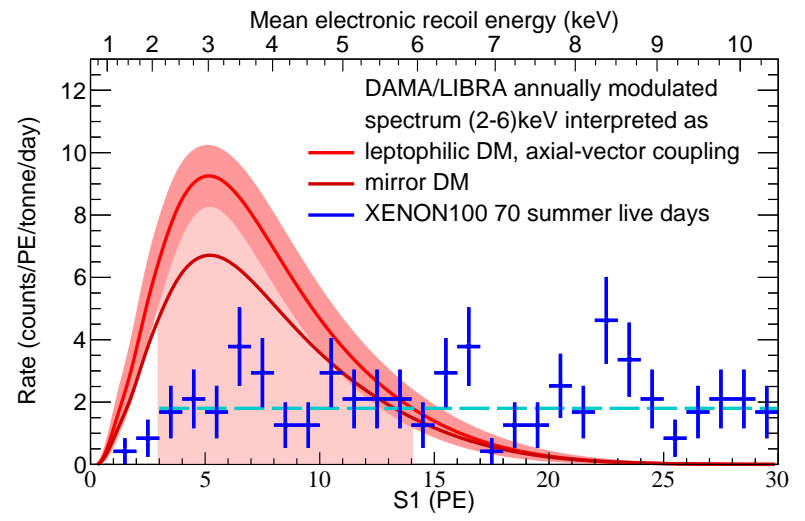

FIG. 3: Fig. 3. Contrasting XENON100 data with DAMA/LIBRA. The DAMA/LIBRA modulated spectrum (red), interpreted as WIMPs scattering through axial-vector interactions, as it would be seen in the XENON100 detector. The $1 \sigma$ band includes statistical and systematic uncertainties. The DAMA/LIBRA modulated spectrum interpreted as luminous dark matter is very similar, whereas the interpretation as mirror dark matter is indicated separately (dark red). The (blue) data points are XENON100 data from the 70 summer live days with their statistical uncertainty. The expected average XENON100 rate is also shown (dashed cyan). The shaded region from (3-14) PE was used to quantify the confidence level of exclusion.

WIMPs and electrons at $4.4 \sigma$ significance level, even considering all events from the well-understood XENON100 background [19] as signal candidates. To be consistent with previous analyses [16], the same data selection cuts were applied. The exclusion remains unchanged if we only impose a minimum set of requirements, namely that events have a single scatter in the fiducial volume with a prompt S1 and delayed S2 signal in the correct energy range. Furthermore, the exclusion stays above $3 \sigma$ confidence level even if we consider a $4.5 \sigma$ downward deviation in the measured data points [26-28] that are used to set the energy scale, or if we set the light yield in xenon to zero below $2.9 \mathrm{keV}$, in contradiction with direct measurement $[27,28]$.

A profile likelihood analysis [30, 31] was performed to constrain the cross section $\sigma_{\chi e}^{0} \equiv G^{2} m_{e}^{2} / \pi$ for WIMPs coupling to electrons through axial-vector interactions. To this end, we drop the assumption of a $100 \%$ modulated rate and use the entire 224.6 live days data set. Fully analogous to [16], we use the same energy range and background likelihood function, derived from calibration data. We do not consider energy depositions below $1 \mathrm{keV}$, the lowest directly measured data point in [27]. The resulting XENON100 exclusion limit (90\% confidence level) is shown (Fig. 4) along with the $1 \sigma / 2 \sigma$-sensitivity bands based on the background-only hypothesis. It excludes cross-sections above $6 \times 10^{-35} \mathrm{~cm}^{2}$ for WIMPs with a mass of $m_{\chi}=2 \mathrm{GeV} / \mathrm{c}^{2}$. This is more than 5 orders of magnitude stronger than the one derived in [11] based on 


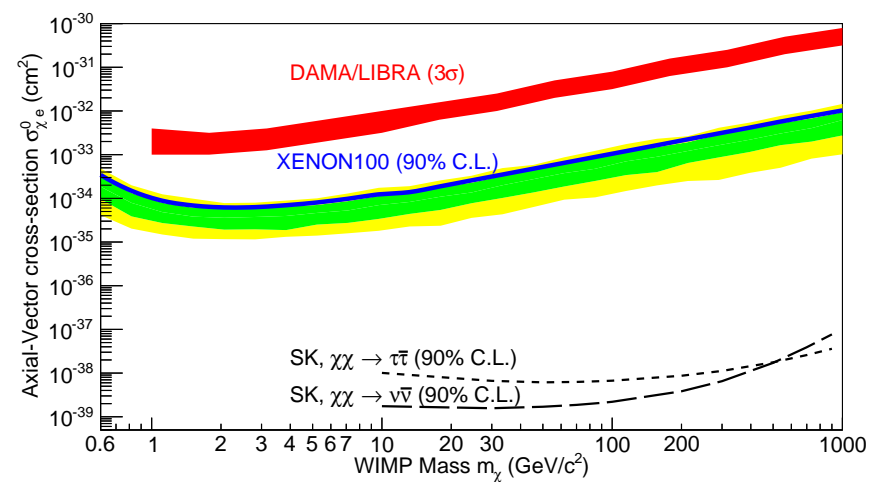

FIG. 4: Fig. 4. Parameter space for WIMPs coupling to electrons through axial-vector interactions. The XENON100 upper limit (90\% confidence level) is indicated by the blue line, along with the green/yellow bands indicating the $1 \sigma / 2 \sigma$ sensitivity. For comparison, we also show the DAMA/LIBRA allowed region (red) and the constraint from Super-Kamiokande (SK) using neutrinos from the Sun, by assuming dark matter annihilation into $\tau \bar{\tau}$ or $\nu \bar{\nu}$, both calculated in [11].

data from the XENON10 detector, completely excludes the DAMA/LIBRA signal, and sets the strongest direct limit to date on the cross section of WIMPs coupling to electrons through axial-vector interactions.For comparison, we also show the DAMA/LIBRA allowed region and the constraint from Super-Kamiokande using neutrinos from the Sun, by assuming dark matter annihilation into $\tau \bar{\tau}$ or $\nu \bar{\nu}$, both calculated in [11]. The XENON100 data completely excludes the DAMA/LIBRA signal and sets the strongest direct limit to date on the cross section of WIMPs coupling to electrons through axial-vector interactions, excluding cross-sections above $6 \times 10^{-35} \mathrm{~cm}^{2}$ for WIMPs with a mass of $m_{\chi}=2 \mathrm{GeV} / \mathrm{c}^{2}$.

Kinematically Mixed Mirror Dark Matter: It has been suggested that multi-component models with light dark matter particles of $\sim \mathrm{MeV} / \mathrm{c}^{2}$ mass might explain the DAMA/LIBRA modulation [32]. A specific example of such a model, kinematically mixed mirror dark matter [33], was shown to broadly have the right properties to explain the DAMA/LIBRA signal via dark matterelectron scattering. In this model, dark matter halos are composed of a multi-component plasma of mirror particles, each with the same mass as their standard model partners. The mirror sector is connected to the normal sector by kinetic mixing of photons and mirror photons at the level of $\sim 10^{-9}$, which provides a production mechanism for mirror dark matter and a scattering channel with ordinary matter. While mirror hadrons would not induce nuclear recoils above threshold, mirror electrons $\left(m_{e}^{\prime}=511 \mathrm{keV} / \mathrm{c}^{2}\right)$ would have a velocity dispersion large enough to induce $\sim \mathrm{keV}$ electronic recoils.

The differential scattering rate of mirror electrons is proportional to $g N n_{e^{\prime}}$, where $g$ is the number of looselybound electrons, assumed to be those with binding en- ergy $<1 \mathrm{keV}$ [33], $N$ is the number of target atoms and $n_{e^{\prime}}$ is the mirror electron density. The detectordependent quantities are $N$ and $g$. In order to compare DAMA/LIBRA directly with XENON100, we apply a constant scaling of $g_{\mathrm{Xe}} / g_{\mathrm{NaI}} \cdot N_{\mathrm{Xe}} / N_{\mathrm{NaI}}=0.89$ to the DAMA/LIBRA spectrum and use the same procedure as in the case of axial-vector coupling: We again consider only the DAMA/LIBRA modulation signal, use the 70 summer live days, model scintillation in liquid xenon as described previously, and simply compare integral counts up to the point where the DAMA/LIBRA signal falls below the expected average XENON100 background data rate (at $13 \mathrm{PE}$ ), without background subtraction. This excludes the DAMA/LIBRA signal as kinematically mixed mirror dark matter at $3.6 \sigma$ confidence level.

Luminous Dark Matter: The third model we consider is Luminous Dark Matter [34], featuring a dark matter particle with a $\sim \mathrm{keV}$ mass splitting between states connected by a magnetic dipole moment operator. The dark matter particle upscatters in the Earth and later deexcites, possibly within a detector, with the emission of a real photon. The experimental signature of this model is a mono-energetic line from the de-excitation photon. A mass splitting $\delta=3.3 \mathrm{keV}$ provides a good fit to the DAMA/LIBRA signal [34] which would be explained as scattering of a real photon from the de-excitation of a $\sim \mathrm{GeV} / \mathrm{c}^{2}$ dark matter particle that is heavy enough to undergo upscattering, but light enough to evade detection in other direct searches.

This signature is independent of the target material; only the sensitive volume affects the induced event rate. As rates are typically given per unit detector mass, scaling to volume is inversely proportional to target density. We thus apply a constant scaling factor to the differential rate in DAMA/LIBRA which is the ratio of the target densities $\rho_{\mathrm{NaI}} / \rho_{\mathrm{Xe}}=1.29$ in order to compare it to XENON100. Proceeding as in the previous two cases, we exclude the DAMA/LIBRA signal as luminous dark matter at $4.6 \sigma$ confidence level. Together with the other two exclusions presented above, this robustly rules out leptophilic dark matter interactions as cause for the DAMA/LIBRA signal.

Acknowledgements: We thank J. Kopp for providing the calculated wave functions and for useful discussions. We gratefully acknowledge support from the National Science Fundation, Department of Energy, Swiss National Science Foundation, Volkswagen Foundation, Bundesministerium fur Bildung und Forschung, Max Planck Gesellschaft, Research Center Elementary Forces and Mathematical Foundations, Foundation for Fundamental Research on Matter, Weizmann Institute of Science, Initial Training Network Invisibles, Fundacao para a Ciencia e a Tecnologia, Region des Pays de la Loire, Science and Technology Commission of Shanghai Municipality, National Natural Science Foundation of China, 
and Istituto Nazionale di Fisica Nucleare. We are grateful to Laboratori Nazionali del Gran Sasso for hosting and supporting the XENON project. XENON data is archived at the Laboratori Nazionali del Gran Sasso.

* Electronic address: mcervant@purdue.edu

$\dagger$ Electronic address: rafael@purdue.edu

¥ Also with Coimbra Engineering Institute, Coimbra, Portugal

$\S$ Present address: IFIC, CSIC-Universidad de Valencia, Valencia, Spain

[1] L. Bergstrom, Dark Matter Evidence, Particle Physics Candidates and Detection Methods, Annalen Phys. 524, 479 (2012), arXiv: 1205.4882.

[2] A. Drukier, K. Freese, D. Spergel, Detecting Cold Dark Matter Candidates, Phys.Rev. D33, 3495 (1986).

[3] K. Freese, M. Lisanti, C. Savage, Annual Modulation of Dark Matter: A Review, Rev.Mod.Phys. 85, 1561 (2013), arXiv:1209.3339.

[4] R. Bernabei et al., Final model independent result of DAMA/LIBRA-phase1, Eur.Phys.J. C73, 2648 (2013), arXiv:1308.5109.

[5] C. Savage, G. Gelmini, P. Gondolo, K. Freese, Compatibility of DAMA/LIBRA dark matter detection with other searches, J.Cosm.Astropart.Phys. 0904, 010 (2009), arXiv:0808.3607.

[6] D. Akerib et al., First results from the LUX dark matter experiment at the Sanford Underground Research Facility, Phys.Rev.Lett. 112, 091303 (2014), arXiv: 1310.8214.

[7] R. Agnese et al., Search for Low-Mass WIMPs with SuperCDMS, Phys.Rev.Lett. 112, 241302 (2014), arXiv: 1402.7137.

[8] Y. Bai, P. J. Fox, Resonant Dark Matter, J.High En.Phys. 0911, 052 (2009), arXiv:0909.2900.

[9] S. Chang, N. Weiner, I. Yavin, Magnetic Inelastic Dark Matter, Phys.Rev. D82, 125011 (2010), arXiv: 1007.4200 .

[10] S. Chang, R. F. Lang, N. Weiner, Effect of Thallium Impurities in the DAMA Experiment on the Allowed Parameter Space for Inelastic Dark Matter, Phys.Rev.Lett. 106, 011301 (2011), arXiv: 1007.2688.

[11] J. Kopp, V. Niro, T. Schwetz, J. Zupan, DAMA/LIBRA and leptonically interacting Dark Matter, Phys.Rev. D80, 083502 (2009), arXiv:0907.3159.

[12] S. Chang, R. Edezhath, J. Hutchinson, M. Luty, Leptophilic Effective WIMPs, Phys. Rev. D90, 015011 (2014), arXiv: 1402.7358.

[13] N. F. Bell, Y. Cai, R. K. Leane, A. D. Medina, Leptophilic Dark Matter with $Z^{\prime}$ interactions, Phys.Rev. D90, 035027 (2014), arXiv: 1407.3001.

[14] E. Aprile et al., Dark Matter Results from 225 Live Days of XENON100 Data, Phys.Rev.Lett. 109, 181301 (2012), arXiv: 1207.5988.

[15] E. Aprile et al., Limits on spin-dependent WIMP-nucleon cross sections from 225 live days of XENON100 data, Phys.Rev.Lett. 111, 021301 (2013), arXiv:1301.6620.

[16] E. Aprile et al., First Axion Results from the XENON100 Experiment, Phys.Rev. D90, 062009 (2014), arXiv: 1404.1455.

[17] E. Aprile et al., The XENON100 Dark Matter Experi- ment, Astropart.Phys. 35, 573 (2012), arXiv:1107.2155.

[18] E. Aprile et al., Analysis of the XENON100 Dark Matter Search Data Astropart.Phys. 54, 11 (2014), arXiv: 1207.3458.

[19] E. Aprile et al., Study of the electromagnetic background in the XENON100 experiment, Phys.Rev. D83, 082001 (2011), arXiv:1101.3866.

[20] R. Bernabei, et al., First results from DAMA/LIBRA and the combined results with DAMA/NaI, Eur.Phys.J. C56, 333 (2008), arXiv:0804.2741.

[21] V. Kudryavtsev, M. Robinson, N. Spooner, The expected background spectrum in NaI dark matter detectors and the DAMA result, Astropart.Phys. 33, 91 (2010), arXiv:0912.2983.

[22] J. Herrero-Garcia, T. Schwetz, J. Zupan, On the annual modulation signal in dark matter direct detection, J.Cosm.Astropart.Phys. 1203, 005 (2012), arXiv: 1112.1627.

[23] E. Aprile et al., Search for Event Rate Modulation in XENON100 Electronic Recoil Data (2015).

[24] R. Bernabei et al., Investigating electron interacting dark matter, Phys.Rev. D77, 023506 (2008), arXiv:0712.0562.

[25] M. Szydagis et al., NEST: A Comprehensive Model for Scintillation Yield in Liquid Xenon, J.Instrum. 6, P10002 (2011), arXiv:1106.1613.

[26] A. Manalaysay et al., Spatially uniform calibration of a liquid xenon detector at low energies using $83 \mathrm{~m}-\mathrm{Kr}$, Rev.Sci.Instrum. 81, 073303 (2010), arXiv:0908.0616.

[27] E. Aprile et al., Measurement of the Scintillation Yield of Low-Energy Electrons in Liquid Xenon, Phys.Rev. D86, 112004 (2012), arXiv:1209.3658.

[28] L. Baudis et al., Response of liquid xenon to Compton electrons down to $1.5 \mathrm{keV}$, Phys.Rev. D87, 115015 (2013), arXiv: 1303.6891.

[29] M. Szydagis, A. Fyhrie, D. Thorngren, M. Tripathi, Enhancement of NEST Capabilities for Simulating LowEnergy Recoils in Liquid Xenon, J.Instrum. 8, C10003 (2013), arXiv: 1307.6601.

[30] G. Cowan, K. Crammer, E. Gross, O. Vitellis, Asymptotic formulae for likelihood-based tests of new physics. Eur.Phys.J. C71, 1554 (2011), arXiv:1007.1727.

[31] E. Aprile et al., Likelihood Approach to the First Dark Matter Results from XENON100, Phys. Rev. D84, 052003 (2011), arXiv: 1103.0303.

[32] R. Foot, H. Lew, R. Volkas, A Model with fundamental improper space-time symmetries, Phys.Lett. B272, 67 (1991).

[33] R. Foot, Mirror dark matter: Cosmology, galaxy structure and direct detection, Int.J.Mod.Phys. A29, 1430013 (2014), arXiv: 1401.3965.

[34] B. Feldstein, P. W. Graham, S. Rajendran, Luminous Dark Matter, Phys.Rev. D82, 075019 (2010), arXiv: 1008.1988.

\section{Supporting Online Material:}

- S1 signal of low-energy electronic recoil events (file1_s1energies.dat)

- Recoil spectra for a $200 \mathrm{GeV} / \mathrm{c}^{2}$ WIMP with axialvector couplings to electrons in $\mathrm{NaI}$ and $\mathrm{Xe}\left(\mathrm{file}{ }_{2}\right.$ axialvectorspectrum.dat) 